\title{
Estudio preliminar de la diversidad genética de perros con fenotipo Poodle en Colombia usando microsatélites
}

\author{
Infante, J. ${ }^{1}$; Bernal, Y. ${ }^{2}$; Acosta, E. ${ }^{3}$; Gómez, L. ${ }^{4}$ y Torres O. ${ }^{2 @}$
}

\begin{abstract}
'Fundación Universitaria Agraria de Colombia - UNIAGRARIA. Bogotá. Colombia. ${ }^{2}$ Facultad de Medicina Veterinaria. Universidad Antonio Nariño. Bogotá. Colombia. ${ }^{3}$ Departamento de Biología. Universidad Nacional de Colombia. Bogotá. Colombia. ${ }^{4}$ Centro Médico Veterinario. Bogotá. Colombia.
\end{abstract}

Palabras ClaVe adicionales

Hardy-Weinberg.

Endogamia.

Heterocigosidad.

Polimorfismo.

\section{RESUMEN}

El objetivo de este estudio fue estimar la variabilidad genética de una muestra poblacional de perros con fenotipo Poodle en Bogotá - Colombia. La variabilidad genética de 81 perros con fenotipo Poodle se estudió mediante el genotipado de cinco loci de microsatélites. La heterocigosidad esperada $\left(\mathrm{H}_{\mathrm{E}}\right)$ para los loci: AHTK253, CXX279, INU030, INU055 y REN162CO4 fue de $0,747,0,788,0,767,0,679$ y 0,751 , respectivamente. La $\mathrm{H}_{\mathrm{E}}$ para el conjunto de la población fue de 0,746. La población de Poodles analizada tuvo una alta diversidad genética y se encontró que no existe una subestructuración poblacional, hecho que desvía el equilibrio HW; con lo que demuestra que la población de Poodle colombiana sea una perfecta candidata para estudios de asociación genética, y de conservación de la biodiversidad de los animales domésticos.

\section{Genetic diversity of the Colombian Poodle dogs using microsatellites - preliminary study}

\section{SUMMARY}

The objective of this study was to estimate the genetic variability of a dog population sample of the Poodle breed from Bogotá - Colombia. The genetic variability of 81 Poodle dogs was studied by genotyping of five microsatellite loci. The expected heterozygosity $\left(\mathrm{H}_{\mathrm{E}}\right)$ of the loci AHTk253, CXX279, INU030, INU055 and REN162C04 was 0.747, 0.788, 0.767, 0.679 and 0.751 , respectively. The $H_{E}$ for the whole population was 0.746 . The Poodle population analyzed showed high genetic diversity, and it was demonstrated that there is no a substructure in that population, which leads to a deviation in the HW equilibrium; thereby demonstrating that the population of Colombian Poodle is a perfect candidate for studies of genetic association, and biodiversity conservation of domestic animals.

\section{INFORMACIÓN}

Cronología del artículo.

Recibido/Received: 10.5.2015

Aceptado/Accepted: 12.6.2015

On-line: 16.9.2015

Correspondencia a los autores/Contact e-mail:

ortorres@uan.edu.co

\section{INTRODUCCIÓN}

Se ha sugerido que el actual número de grupos genéticamente diferenciado de razas de perros podría ser aún más grande que el número de razas existentes, como en el caso del Poodle, en el cual, los análisis genéticos han mostrado una subestructura poblacional, que probablemente podría abarcar múltiples subgrupos genéticamente divergentes (Björnerfeldt et al., 2008); y puesto que la clasificación de los perros en la actualidad se basa en las características fenotípicas, puede darse el caso de que no exista homogeneidad genética dentro de la mismo grupo racial. El caracterizar genéticamente al perro doméstico, tiene gran importancia, no solo desde el ámbito de la conservación de la biodiversidad de los animales domésticos, la medicina veterinaria; sino también de la medicina humana (Kim et al., 2012). La selección de las razas de perros es tan estricta, que cada población de estos individuos tiende a representar una población cerrada con altos niveles de homogeneidad fenotípica (Parker et al., 2006), lo que ha conducido también a una alta selección de un número de enfermedades; permitiendo que esta especie pueda ser útil en la identificación de marcadores genéticos asociados a la presencia de dichas patologías (Cadieu y Ostrander, 2007). Adicionalmente, las patologías en el perro a menudo imitan las enfermedades humanas muy estrechamente. Del total de enfermedades genéticas comunes al perro y el humano, aproximadamente el $46 \%$ se producen principalmente en una o unas pocas razas; lo que los hace un modelo ideal para mapear los genes de de enfermedades del humano (Parker y 
Ostrander, 2005); pero de nuevo, partiendo del hecho de que los estudios genéticos de asociación, dependen de la uniformidad genética de los individuos que hacen parte del estudio (Sutter et al., 2004), es necesario determinar si existe homogeneidad dentro del grupo de los individuos evaluados. La presente investigación realiza un doble aporte a este respecto: 1) de manera preliminar se describe la diversidad genética de esta población, y 2) se inician los estudios que permitirán implementar al Poodle como modelo para estudios de asociación genética.

\section{MATERIAL Y MÉTODOS}

\section{POBLACIÓN DE ESTUDIO}

La muestra se conformó de 81 caninos con fenotipo Poodle de tamaño mediano $(35-45 \mathrm{~cm})$, seleccionados aleatoriamente y sin relación de parentesco en la ciudad de Bogotá. Los perros fueron asignados al morfotipo Poodle si cumplian con por lo menos el $80 \%$ de las características del prototipo dadas por la Federación Cinológica Internacional (http: / / www.fci.be/Nomenclature/Standards/172g09-en.pdf).

\section{SELECCIÓN DE MARCADORES}

Se escogieron cinco marcadores STR: AHTK253, CXX279, INU030, INU055 y REN162C04; recomendados por la Sociedad Internacional de Genética Animal - ISAG (wwww.isag.us/Docs/.../2005ISAGPanelDOG.pdf).

\section{GENOTIPIFICACIÓN}

el ADN fue extraído según el método descrito por Miller et al. (1988) a partir de $3 \mathrm{ml}$ de sangre total tomada en tubo venojet tapa lila, por punción de la vena cefálica. Los STR fueron amplificados mediante PCR; y la reacción de amplificación de cada muestra se realizó a un volumen final de $10 \mu \mathrm{l}$ cada una, que incluían una multiplex de los cinco loci. La mezcla para la PCR incluyó 1x de TaqMan ${ }^{\circledR}$ Universal PCR Master Mix buffer (Applied Biosystem, Foster City, California) y $1 \mu \mathrm{l}$ ADN molde. El protocolo de amplificación incluyó un paso de desnaturalización inicial del $\mathrm{ADN}$ a $95^{\circ} \mathrm{C}$ por 10 minutos, seguido por 40 ciclos de 60 segundos de desnaturalización a $95^{\circ} \mathrm{C}, 30$ segundos de anillamiento a $58^{\circ} \mathrm{C}$, y 45 segundos de extensión a $72^{\circ} \mathrm{C}$ usando un equipo Eco Real-Time PCR System ${ }^{\circledR}$ (Illumina, Inc., San Diego, CA, USA). La discriminación alélica de los amplificados se realizó por electroforesis capilar en un secuenciador automático ABI PRISM 3500 (Applied Biosystems) incluyendo el marcador de peso molecular - GeneScan ${ }^{\text {TM }} 600$ LIZ $^{\circledR}$ (Applied Biosystems). El genotipado fue identificado usando el software GeneMarker v.2.6.3 (Softgenetics, State College, PA, USA).

\section{ANÁLISIS ESTADÍSTICO}

Se utilizó el programa GenAlEx v.6.3 (Peakall y Smouse, 2006) para calcular las frecuencias alélicas, número de alelos detectados $\left(\mathrm{N}_{\mathrm{A}}\right)$, número medio de alelos por locus (NMA), heterocigosis observada $y$ esperada $\left(\mathrm{H}_{\mathrm{O}} \mathrm{y} \mathrm{H}_{\mathrm{E}}\right)$. El índice de polimorfismo (PIC) se calculó con el programa PICcalc (Nagy et al., 2012). El programa FSTAT v.2.9.3.2 (Goudet, 1995) se utilizó para detectar las desviaciones del equilibrio Hardy-Weinberg (HW), además de los F-estadísticos, los cuales se
Tabla I. Parámetros de diversidad genética de la población de Poodle (Genetic diversity parameters of the Poodle population).

\begin{tabular}{lcccccc}
\hline Locus & $\mathrm{N}$ & $\mathrm{N}_{\mathrm{A}} / \mathrm{NMA}$ & $\mathrm{Ho}$ & $\mathrm{H}_{\mathrm{E}}$ & $\mathrm{F}_{\mathrm{IT}}$ & $\mathrm{PIC}$ \\
\hline AHTK253 & 81 & 8 & 0,654 & 0,679 & $0,037^{\text {ns }}$ & 0,704 \\
CXX279 & 81 & 8 & 0,691 & 0,767 & $0,099^{\text {ns }}$ & 0,751 \\
INU030 & 81 & 6 & 0,691 & 0,747 & $0,075^{\text {ns }}$ & 0,724 \\
INU055 & 81 & 8 & 0,778 & 0,788 & $0,013^{\text {ns }}$ & 0,637 \\
REN162C04 & 69 & 6 & 0,754 & 0,751 & $-0,003^{\text {ns }}$ & 0,705 \\
Total & & 7,2 & 0,714 & 0,746 & $0,044^{\text {ns }}$ & \\
IDE & & 0,97 & 0,045 & 0,036 & &
\end{tabular}

$\mathrm{N}=$ tamaño muestral; $\mathrm{NA}=$ número de alelos; $\mathrm{NMA}=$ número medio de alelos para la población total; $\mathrm{HO}=$ heterocigosis observada; $\mathrm{HE}=$ heterocigosis esperada; $\mathrm{FIT}=(-)$ exceso o (+) déficit de heterocigotos; $\mathrm{PIC}=$ índice de polimorfismo; $n s=$ no significativo $(p<0,05)$; $\pm \mathrm{DE}=$ desviación estándar.

calcularon mediante los estimadores de Weir y Cockerham (1984). Para determinar si existe subestructuración poblacional se utilizó el programa STRUCTURE v.2.3.3.(Pritchard et al., 2000). Se evaluó el número de subpoblaciones posibles $(\mathrm{K})$ a través de un rango para $\mathrm{K}$ de 1 a 4. La probabilidad (máxima verosimilitud) de los diferentes valores fue evaluado asumiendo el Ln P(D).

\section{RESULTADOS Y DISCUSIÓN}

Los 5 loci fueron polimórfícos y el número total de alelos detectados fue de 36 , con un número promedio de 7,2. Con respecto al índice de polimorfismo (PIC) se observa que para la población colombiana de Poodle todos los loci fueron muy informativos (PIC>0,637). La heterocigosis esperada para el conjunto de la población fue de 0,746. La población presentó un déficit de heterocigotos según el coeficiente de endogamia $\left(F_{\text {IT }}: 0,044\right)$. El valor del $F_{\text {IT }}$ tiene una $p>0,05$, con lo que se infiere que la población se encontró dentro del equilibrio Hardy-Weinberg, lo que infiere que no existe subestructuración genética en la población (tabla I).

La no subestructuración se respalda mediante los valores de Ln P(D), los cuales mostraron que el valor promedio más probable de K es 1 (figura 1A). De igual manera al calcular las frecuencias porcentuales más probables de cada individuo para cada cluster se observa una gran homogeneidad entre los genomas de los individuos cuando se realizó el análisis para $\mathrm{K}=2$ con el programa STRUCTURE (figura 1B).

En este estudio se muestrearon individuos con fenotipo Poodle que aunque no se encuentran dentro de un pedigrí, ya que no existe una asociación para esta raza, y tampoco se registran ejemplares con dicho fenotipo en Colombia (http: / / www.accc.com.co), sí se encontró un $\mathrm{F}_{\mathrm{IT}}$ positivo $\left(\mathrm{F}_{\mathrm{IT}}: 0,044\right)$ que refleja un cierto grado de endogamia. No obstante, hay que tener en cuenta que entre las diferentes razas de perros es habitual encontrar valores positivos para el $\mathrm{F}_{\mathrm{IT}}$ debido al alto grado de selección al que fueron sometidos (Wictum et al., 2013), que no sería el caso en este estudio por las razones antes mencionadas. Cuando nos referimos a que la población de este estudio posee cierto grado de 
A

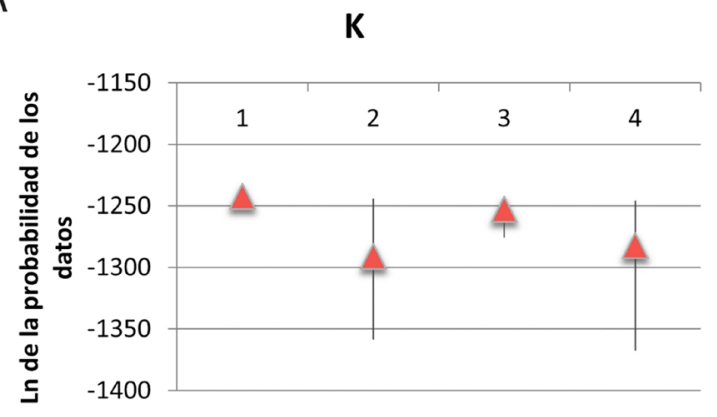

B

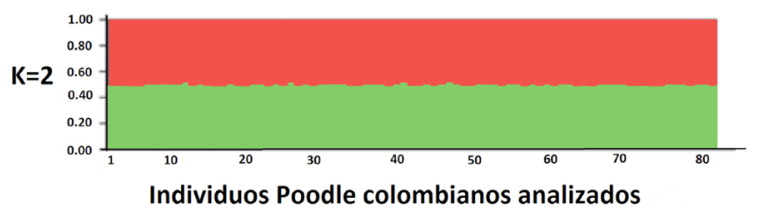

Figura 1. Análisis de la estructura de la población de estudio, utilizando STR mediante el programa STRUCTURE. (A) Gráfico del comportamiento del logaritmo de probabilidad Ln p(D) en función del número de subpoblaciones $\mathrm{K}$, indicando 1 como el número óptimo de clusters genéticos. (B) Representación gráfica en barras mostrando la distribución del genoma ancestral (ancestría) con una asignación de individuos de $\mathrm{K}=2$, indicando que el genoma no se ha diversificado en la población de Poodle colombiano (Analysis of the structure of the studied Poodle population by using STR through the STRUCTURE software. (A) Graphic of the probability logarithm behavior $\operatorname{Ln} p(D)$ in function of the number of subpopulations $\mathrm{K}$ that indicates 1 as the optimal number of genetic clusters. (B) Bar graph that shows the distribution of the ancestral genome (ancestry) with an individual assignation of $\mathrm{K}=2$, that indicates that the genome has not diversified in the Colombian Poodle population).

endogamia, queremos decir, que aunque los individuos no tienen un coeficiente alto de coancestría, sí están asociados ancestralmente. El coeficiente de endogamia en Poodle ha sido mayor en trabajos anteriores ( $\mathrm{F}_{\mathrm{IT}}$ : 0,072 (Björnerfeldt et al., 2008); $\mathrm{F}_{\mathrm{IT}}$ : 0,080 (Pedersen y Kennedy, 2012) que el reportado en el presente trabajo. Esta reducción del coeficiente de endogamia encontrada, ha sido generada tal vez por el ingreso de los nuevos alelos de los cruces con perros de distintas razas. La consecuencia de lo encontrado es, que aunque en Colombia aún no existen razas autóctonas de caninos, es muy probable que haya poblaciones con las características morfológicas y genéticas para ser reconocidas con el estatus de raza como es el caso de la población en mención, así como otras.

Todos los marcadores moleculares utilizados en el presente trabajo mostraron valores elevados de polimorfismo (PIC>0,637), objetivo primordial para disminuir el sesgo en el uso de los diferentes estadísticos genético-poblacionales; además, los valores son similares a los reportados en otras razas caninas (Radko y Slota, 2008). Los datos estimados para la diversidad genética de la población de estudio $\left(\mathrm{H}_{\mathrm{E}}\right.$ : 0,746) se encuentra semejante a la media calculada para 25 razas con más de 1000 individuos reportado por Wictum et al. (2013) usando otros marcadores de tipo STR
$\left(\mathrm{H}_{\mathrm{E}}: 0,755\right)$. Sin embargo, la población en el presente estudio presenta valores de heterocigosidad esperada mayores que los reportados por diferentes autores para la raza Poodle (Perdersen y Kennedy, 2012). Este aumento de la diversidad genética puede ser el resultado de la mezcla que debió ocurrir entre los Poodle puros que entraron en Colombia con las demás poblaciones caninas existentes, además de las poblaciones criollas (Asch et al., 2013). El resultado de dichos cruces debió añadir nuevos alelos de las demás poblaciones, con lo que se esperaría que en las subsiguientes generaciones de Poodle la frecuencia de dichos alelos aumentara y se mantuvieran estables. Dicha estabilidad se constata con el estadístico HW, donde todos los loci se encuentran en equilibrio.

Contrario a lo observado en el presente estudio, Björnerfeldt et al. (2008) vieron que al analizar a los Poodle como una sola población, esta se muestra en desequilibrio HW, demostrando tal vez, que la discriminación de los tamaños del Poodle al realizar los cruces podría tener un efecto sobre dicho estadístico. Otros trabajos, como el de Short et al. (2007), demuestran queal separar los Poodle por tamaño, los genes estudiados también se encuentran en su mayoría dentro del equilibrio HW. Para el presente estudio no se discriminaron los individuos en grupos por tamaño o color; sin embargo se demostró que no existen probables subestructuras, siendo demostrado por el programa STRUCTURE, por lo que no vale la pena realizar análisis a este respecto. Adicionalmente, se demuestra que la población de estudio no está sufriendo procesos fuera de los supuestos para el equilibrio HW, como lo son deriva genética, migración o selección; y de manera importante se comporta como una unidad panmíctica con la misma probabilidad de apareamiento de los individuos (Short et al., 2007).

\section{CONCLUSIONES}

Dado que no se encontró subestructuración en la población muestreada, denotando una gran uniformidad genética, y que además, la población se encuentra bajo equilibrio Hardy-Weinberg, se propone a los perros con fenotipo Poodle colombianos como candidatos para realizar estudios de asociación genética. Adicionalmente, esta población podría tener características genéticas propias para ser reconocida como raza en Colombia, con lo que aportaría diversidad genética dentro de la familia de cánidos domésticos a nivel local y mundial.

\section{BIBLIOGRAFÍA}

Asch, B.; Zhang, A.; Oskarsson, M.C.R.; Klütsch, C.F.C.; Amorim, A. and Savolainen, P. 2013. Pre-Columbian origins of native American dog breeds, with only limited replacement by European dogs, confirmed by mtDNA analysis. PR Soc Lond B Bio, 280: 1766.

Bï̈rnerfeldt, S.; Hailer, F.; Nord, M. and Vilà, C. 2008. Assortative mating and fragmentation within dog breeds. BMC Evol Biol, 8: 28.

Cadieu, E. and Ostrander, E.A. 2007. Canine genetics offers new mechanisms for the study of human cancer. Cancer Epidem Biomar, 16: $2181-2183$.

Goudet, J. 1995. FSTAT (Version 1.2): A Computer program to calculate F-statistics. J Hered, 86: 485-486. 
Kim, R.N.; Kim, D.S.; Choi, S.H.; Yoon, B.H.; Kang, A.; Nam, S.H.; Kim, D.W.; Kim, J.J.; Hong Ha, J.H.; Toyoda, A.; Füiyama, A.; Kim, A.; Kim, M.Y.; Park, K.H.; Lee, K.S. and Park, H.S. 2012. Genome analysis of the domestic dog (Korean Jindo) by massively parallel sequencing. DNA Res, 19: 275-288.

Miller, S.A.; Dykes, D.D. and Polesky, H.F. 1988. A simple salting out procedure for extracting DNA from human nucleated cells. Nucleic Acids Res, 16: 1215.

Nagy, S.; Poczai, P.; Cernák, I.; Gorii, A.M.; Hegedüs, G. and Taller, J. 2012. PICcalc: An online program to calculate polymorphic information content for molecular genetic studies. Biochem Genet, 50: 670-672.

Parker, H.G. and Ostrander, E.A. 2005. Canine genomics and genetics: Running with the Pack. PLoS Genet, 1: e58.

Parker, H.G.; Sutter, N.B. and Ostrander, E.A. 2006. 9 Understanding genetic relationships among purebred dogs: The PhyDo project. Cold Spring Harbor M, 44: 141-157.

Peakall, R. and Smouse, P.E. 2006. Genalex 6: genetic analysis in Excel. Population genetic software for teaching and research. Mol Ecol Notes, 6: 288-295.
Pedersen, N.C. and Kennedy, L. J. 2012. A genetic comparison of standard and miniature poodles based on DLA class II haplotypes. http:// www.vetmed.ucdavis.edu/ccah/local-assets/pdfs (06/04/2015).

Pritchard, J.K.; Stephens, M. and Donnelly, P. 2000. Inference of population structure using multilocus genotype data. Genetics, 155: 945-959.

Radko, A. and Slota, E. 2008. Application of 19 microsatellite DNA markers for parentage control in Borzoi dogs. Pol J Vet Sci, 12: 113-117.

Short, A.D.; Kennedy, L.J.; Barnes, A.; Fretwell, N.; Jones, C.; Thomson, W. and Ollier, W. E. 2007. Hardy-Weinberg expectations in canine breeds: Implications for genetic studies. J Hered, 98: 445-451.

Sutter, N.B.; Eberle, M.A.; Parker, H.G.; Pullar, B.J.; Kirkness, E.F.; Kruglyak, L. and Ostrander, E.A. 2004. Extensive and breed-specific linkage disequilibrium in Canis familiaris. Genome Res, 14: 2388-2396. Weir, B.S. and Cockerham, C.C. 1984. Estimating F-statistics for the analysis of population structure. Evolution, 38: 1358.

Wictum, E.; Kun, T.; Lindquist, C.; Malvick, J.; Vankan, D. and Sacks, B. 2013. Developmental validation of DogFiler, a novel multiplex for canine DNA profiling in forensic casework. Forensic Sci Int-Gen, 7: 82-91. 